\title{
RECUPERO DE COSTOS EN UN HOSPITAL MILITAR DE ARGENTINA
}

\author{
Carlos Atilio Glerean (•) \\ Milva Geri ( $\cdot \cdot)$ \\ María Eugenia Elorza $(\cdots)$ \\ Instituto de Investigaciones \\ Económicas y Sociales del Sur \\ Universidad Nacional del Sur / CONICET
}

\begin{abstract}
RESUMEN
El recupero de costos de la seguridad social constituye una fuente de financiamiento de los hospitales públicos argentinos. Los hospitales militares también pueden recuperar los costos aunque mediante un proceso con diferente con limitaciones. Se propuso evaluar la factibilidad de mejorarlo a partir de: i) describir el proceso de recupero, ii) cuantificar y caracterizar los costos no recuperados e iii) identificar los problemas e indagar alternativas de mejora. Se revisó la normativa sobre recupero de costos y se caracterizó la facturación por internaciones de pacientes no beneficiarios de la aseguradora militar durante 2016-2018. Se describieron las dificultades y propusieron mejoras que surgen de entrevistas a los actores claves. Se comprobó un alto índice de incobrabilidad y demora en la cadena de pago y una baja incidencia de los recursos recuperados en el financiamiento del hospital. Se evidenció la necesidad de establecer acuerdos formales entre el hospital y las entidades proveedoras y financiadoras de la región. Estos posibilitarían el aprovechamiento regional de la capacidad instalada ociosa en una institución hospitalaria de alta complejidad.
\end{abstract}

\section{PALABRAS CLAVE:}

Costos de hospital, Hospitales militares, Argentina.

(•) E-mail: carlosglerean@gmail.com

(•.) E-mail: mgeri@iiess-conicet.gob.ar

(‥)E-mail: meugilorz@hotmail.com

\section{ABSTRACT}

The recovery of social security costs constitutes a source of financing for argentine public hospitals. Military hospitals can also recover the costs, although through a different process with limitations. It was proposed to evaluate the feasibility of improving it by: i) describe the recovery process, ii) quantify and characterize the not recovered costs and iii) identify the problems of the process and investigate alternatives for improvement. The cost recovery regulations were reviewed and the billing for hospitalizations of non-beneficiary patients of the military insurer during 2016-2018 was characterized. Difficulties were described and improvements suggested by interviews with key actors were proposed. A high rate of uncollectibility and delay in the payment chain and a low incidence of the resources recovered in hospital financing were verified. The need to establish formal agreements between the hospital and the provider and financing entities of the region was evident. These would allow the regional use of idle capacity in a highly complex hospital institution.

\section{KEY WORDS:}

Hospital Costs, military hospitals, Argentina.

RECEPCIÓN: 24/05/20

ACEPTACIÓN FINAL: 11/09/20 


\section{IntRoducción}

El sistema de salud argentino se caracteriza por la separación entre los tres subsectores que lo componen: el público, el privado y el de la seguridad social. El público está orientado hacia la población sin cobertura de salud o sin recursos económicos y se divide en nacional, provincial y municipal; el privado está representado por las entidades de medicina privada prepaga y los usuarios con capacidad de pago para financiar sus gastos de salud; mientras que el de la seguridad social está compuesto por las obras sociales sindicales y/ o provinciales y por el Instituto Nacional de Servicios Sociales para Jubilados o Pensionados (conocido como PAMI) (Belló y Becerril-Montekio, 2011).

Estos subsectores tienen diferentes lógicas de funcionamiento y están escasamente coordinados lo cual afecta negativamente la eficiencia en el uso de los recursos asignados al sector, así como también la equidad en el financiamiento y en el acceso a los servicios de salud (Centrangolo, 2014).

Entre los problemas vinculados al financiamiento, derivados de las múltiples interacciones existentes entre los agentes que conforman el sistema de salud fragmentado, se destacan los subsidios cruzados. Este concepto se emplea para describir diversas situaciones que dan lugar a una transferencia de recursos entre los distintos subsectores de salud. En general, se refieren a los casos 
donde actores del sector público (del nivel de gobierno nacional, provincial o municipal) financian las prestaciones médico-sanitarias brindadas en los proveedores públicos a pacientes con cobertura de obra social o empresa de medicina prepaga. Si bien existen otras modalidades de subsidios cruzados, este subsidio indirecto otorgado por el sector público a los otros dos subsectores constituye una importante causa de ineficiencia e inequidad del sistema de salud $\operatorname{argentino}^{1}$ (Tobar, Olaviaga y Solano, 2012).

Para responder a esta problemática, se creó un nomenclador nacional de precios de prestaciones médico-sanitarias, del cual pueden valerse los hospitales públicos de gestión descentralizada ${ }^{2}$ para facturar sus servicios a empresas de medicina prepaga y obras sociales sindicales, generando así recursos adicionales a los provenientes del Estado. Dicho nomenclador consiste en un listado actualizado de precios de prácticas médicas y funciona como instrumento de referencia para el establecimiento de tarifas (Boletín Oficial de la República Argentina, 2000).

Pese a la existencia de este mecanismo de recupero de costos hospitalarios frecuentemente los montos recuperados por las instituciones públicas son inferiores a los costos de producción. Moscoso y Clark (2010) encuentran que el costo promedio de atención de pacientes beneficiarios de algunas obras sociales supera los montos pagados por tales aseguradoras a la institución hospitalaria, reduciendo los recursos disponibles para la atención población sin cobertura de salud. Aleixechuk, Clark y Temporelli (2013) verifican que el valor recuperado por un hospital municipal descentralizado en concepto de cápitas de PAMI resulta inferior al costo de atención de sus afiliados, comprometiendo los recursos disponibles para financiar la atención de los pacientes sin cobertura social y/ o con recursos insuficientes.

El Hospital Naval de Puerto Belgrano (en adelante HNPB), es un hospital del ámbito nacional que está situado en la Base Naval Puerto Belgrano (localizada en la ciudad de Punta Alta, Provincia de Buenos Aires, Argentina) y pertenece al subsistema de salud de la Armada Argentina (dependiente del Ministerio de Defensa del Estado Nacional). Este subsistema brinda servicios médico-hospitalarios a través de tres efectores de salud distribuidos estratégicamente en el territorio argentino

El HNPB se encuentra gerenciado por un Director General con rango militar (Capitán de Navío de orientación Médico) y tres Subdirecciones subordinadas (Asistencial, Administrativa y Operativa). Tiene la misión de ser capaz de responder de manera adecuada y oportuna a las necesidades de salud del personal militar y civil y a sus familiares, a los afiliados a la obra social de las Fuerzas Armadas y 
a la comunidad en general en situación de catástrofe. Ofrece servicios de varias especialidades y funciona en los niveles I, II y III de complejidad asistencial.

Su población objetivo alcanza aproximadamente a 40.000 usuarios, que incluyen al personal militar y civil de la Armada Argentina (y sus familiares) y los afiliados al Instituto de Obras Sociales de las Fuerzas Armadas (en adelante IOSFA). Asimismo, brinda atención a pacientes que no son beneficiarios de IOSFA e ingresan por urgencias y/o emergencias, siendo obligatoria su atención en forma inmediata, independientemente de la cobertura de salud o su capacidad de pago. De esta manera, el HNPB integra una red regional de prestadores públicos de distinto nivel de complejidad en conjunto con un Hospital Municipal y un Hospital Interzonal. Mientras el primero, localizado en la misma ciudad que el HNPB, se financia con fondos públicos del nivel municipal de gobierno, el segundo se financia con fondos del nivel provincial y se encuentra localizado en la ciudad de Bahía Blanca, ubicada a $28 \mathrm{~km}$ de distancia.

Está previsto que el HNPB recupere los costos de la atención brindada a pacientes del área de influencia que no son beneficiarios de IOSFA. Sin embargo, su mecanismo de recupero de costos difiere del empleado por el resto de los hospitales financiados públicamente. En tal sentido, el HNPB debe cumplir con la Ley 24.156 de Administración Financiera del Sector Público Nacional, la cual exige la firma de contratos $\mathrm{y} /$ o convenios con las instituciones a las cuales presta servicios (Boletín Oficial de la República Argentina, 2000).

Pese a que el HNPB factura los servicios brindados a pacientes no beneficiarios de IOSFA según su cobertura de salud, tanto las obras sociales, empresas de medicina prepaga o aseguradoras de riesgo del trabajo como los hospitales públicos que deberían atenderlos, cuentan con total discreción para decidir el tiempo y la forma de pago debido a la ausencia de convenios o contratos formales específicos, limitando la capacidad del HNPB para recuperar los costos de las prestaciones brindadas. Al mismo tiempo, la inexistencia de acuerdos formales impide que las instituciones responsables de brindar cobertura de salud a los pacientes no beneficiarios de IOSFA en la ciudad donde se ubica este hospital aprovechen la capacidad instalada del HNPB que podría ofrecerles una amplia cartera de servicios de alta complejidad.

Por su parte, los pacientes no beneficiarios de IOSFA podrían ahorrarse los costos de traslado a los centros de atención localizados en la ciudad vecina para recibir prestaciones que no están disponibles en la ciudad del HNPB. De esta manera, el establecimiento de convenios que faciliten el mecanismo de recupero de costos no solo implicaría un beneficio para el HNPB, sino también para las instituciones de la región que prestan servicios para sus afiliados o usuarios. 
Por lo expuesto, adquiere relevancia el análisis del mecanismo de recupero de costos del HNPB. La identificación de la magnitud del problema, así como de las fallas del proceso administrativo, serían de utilidad para definir los lineamientos de eventuales contratos de prestación de servicios en caso de que fuera deseable y factible celebrarlos.

El objetivo general del trabajo es recopilar información de utilidad para sugerir propuestas de mejora del sistema de recupero de costos del HNPB. Los objetivos específicos son: i) describir el proceso de recupero de costos del HNPB, ii) cuantificar y caracterizar la facturación por internación de pacientes no beneficiarios de IOSFA entre enero 2016 y diciembre 2018 y iii) identificar problemas y propuestas de mejora del proceso de recupero de costos según distintas perspectivas.

\section{MÉTODOS}

Las tareas realizadas para abordar cada uno de los objetivos específicos fueron:

i) Para "Describir el proceso de recupero de costos del HNPB»:

a) se detalló brevemente la estructura de funcionamiento del HNPB y se revisó la normativa pertinente que debe cumplirse a los efectos de recuperar los costos en el HNPB ${ }^{3}$.

b) se representó el procedimiento de recupero de costos del HNPB, diferenciando la etapa de facturación y de gestión de las deudas, mediante un diagrama de flujo.

ii) Para "Cuantificar y caracterizar la facturación por internación de pacientes no beneficiarios de IOSFA entre enero 2016 y diciembre 2018":

a) se registró el valor de las siguientes variables asociadas a la facturación de los pacientes no beneficiarios de IOSFA internados en el HNPB durante enero de 2016 y diciembre de 2018: i) fecha de la prestación, ii) condición de cobertura de salud, iii) tipo de cobertura (nombre de la obra social o empresa de medicina prepaga), iv) área o servicio que presta la asistencia, v) valor a facturar (en pesos argentinos), vi) estado de la facturación (pudiendo tomar los valores: SI-NO), vii) fecha de facturación, viii) estado del cobro a noviembre 2019 (pudiendo tomar los valores: SI-NO); ix) fecha de cobro, x) demora desde la fecha de facturación hasta la fecha de pago (en días).

b) se calculó el monto total anual facturado por el HNPB en concepto 
de internaciones de pacientes no beneficiarios de IOSFA. Los montos resultantes se compararon con el presupuesto total del HNPB mediante el cálculo de proporciones.

c) se evaluó la demora en el cobro de las prestaciones facturadas en el período, medida como el tiempo (en días) que transcurre desde la fecha de facturación hasta la fecha de pago, discriminándose por tipo de deudor (institucional o particular), según corresponda.

d) se estableció el incumplimiento de las deudas por año del periodo a través del cálculo de un índice de incobrabilidad de las prestaciones considerando aquellas que a noviembre-2019 permanecían sin cobrar del total de facturaciones anuales; discriminándose según tipo de deudor (institucional o particular).

La información fue provista por la Oficina de Facturación a Ajenos a Obras Sociales del HNPB.

iii) Para «Identificar problemas y propuestas de mejora del proceso de recupero de costos según distintas perspectivas»:

a) se recolectó información acerca de las fortalezas y debilidades del proceso administrativo de recupero de costos a partir de entrevistas a los administrativos a cargo de la Oficina de Facturación a Ajenos a Obras Sociales.

b) se indagó la posibilidad de celebrar contratos de prestación entre el HNPB y las instituciones a las que brinda servicios de internación con mayor frecuencia, a partir de entrevistas a los responsables administrativos o médicos de las mismas.

\section{RESULTADOS}

3.1 PROCESO DE RECUPERO DE COSTOS EN EL HNPB

La subdirección administrativa del HNPB es una de las tres subdirecciones subordinadas a la dirección general del hospital y tiene por objetivo planificar, organizar, coordinar, gestionar y evaluar la utilización de los recursos financieros y la administración de los recursos presupuestarios. Dentro de ella se encuentra la Oficina de Facturación a Ajenos a Obras Sociales (en adelante AOS), cuyas tareas se vinculan al cobro de prestaciones médico-asistenciales brindadas a pacientes ajenos al IOSFA. Estos pueden ser beneficiarios de cualquier prepaga u obra social, incluyendo PAMI, o no poseer ningún tipo de cobertura de salud. 
La posibilidad de recuperar costos está regulada por la Ley 24.156, cuyo objetivo es garantizar la aplicación de los principios de regularidad financiera, legalidad, economicidad, eficiencia y eficacia en la obtención y aplicación de los recursos públicos.

Actualmente el HNPB genera recursos propios a través del cobro de prestaciones brindadas a los pacientes no beneficiarios a IOSFA, sin la debida realización de convenios que exige dicha normativa. Las principales tareas inherentes a la AOS para el cobro de las prestaciones en cumplimiento de la Disposición de Salud $N^{\circ} 13$ de Fundación Sanidad Naval Argentina (en adelante FUSANA), (FUSANA. 2016) incluyen:

a) admitir y cotejar los datos de los pacientes ajenos a IOSFA, a través del documento de identidad, carnet de la obra social y/o página web del padrón de afiliados.

b) discriminar, seleccionar y recopilar las prestaciones, revisando y corrigiendo errores en la documentación comprobatoria de cobro, sean tickets fiscales o formularios de reconocimiento de deuda.

c) cargar los datos en el módulo ventas según su categoría fiscal (exento o gravado) e imprimir el ticket fiscal a entregar al paciente, priorizando el cobro por transferencia o depósito bancario.

d) recopilar toda la documentación justificativa de cobro (notificación, comprobantes de fax o correo electrónico, cartas documento, documentación comprobatoria solicitada) para el caso de facturaciones a obras sociales, empresas de medicina prepaga, hospitales y/o centros de atención de la región, etc.

e) presentar y entregar la liquidación firmada y los antecedentes que acompañarán a la factura, por correo o personalmente.

f) confeccionar notas de crédito al recibirse rechazos o débitos producidos sobre la facturación emitida y comunicar a la FUSANA.

La Ley 24.156 establece que los recursos cobrados deben ingresar a las cuentas de recursos de afectación específica y no pueden destinarse directamente al pago de gastos corrientes. Es por ello que los fondos resultantes de los cobros deben depositarse en las cuentas que operen las tesorerías centrales que administran las jurisdicciones y la administración nacional. En este sentido, el Decreto 2.629/1992 establece la obligatoriedad de remitir a la Contaduría General de la Nación las comunicaciones periódicas reglamentarias respecto a las recaudaciones efectuadas, los compromisos asumidos, los gastos devengados y los pagados con indicación de las imputaciones presupuestarias, 
como así también el ingreso a rentas generales de los saldos sobrantes en los recursos con afectación específica al cierre del ejercicio (Boletín Oficial de la República Argentina, 1992).

Esto implica que, a diferencia de los Hospitales Públicos de Gestión Descentralizada, los Hospitales Militares no pueden disponer directamente de los recursos provenientes de sus ingresos por el cobro de las prestaciones brindadas, lo cual genera un desincentivo a invertir esfuerzos en el proceso administrativo de recupero de costos. Para subsanarlo, la FUSANA, fundación sin fines de lucro creada en 1990, celebró un convenio con la Armada para colaborar con el aprovechamiento integral de la oferta de servicios de salud de los Hospitales Navales. Ese aprovechamiento se plasma a través de la utilización, por parte de la FUSANA de la capacidad remanente de tales nosocomios. Esto comprende el ofrecimiento de los servicios médicos asistenciales disponibles, la facturación a terceros de los servicios prestados y la percepción de los pagos correspondientes. La Armada Argentina, por intermedio del HNPB, imputa a la FUSANA la totalidad de los gastos ocasionados por las prestaciones brindadas a pacientes no IOSFA y la FUSANA destina estos fondos a la adquisición de equipamiento y optimización de la calidad de atención del HNPB (Boletín Oficial de la República Argentina, 2015).

La articulación entre el HNPB y la FUSANA permite una administración directa de los ingresos que se perciben en concepto de prestaciones facturadas y genera un incentivo al recupero de costos hospitalarios. La facturación se realiza en base a valores fijados por la Dirección General del HNPB mediante un tarifario que tiene formato de nomenclador de prácticas médicas. En particular, en el HNPB estos valores se establecen considerando un promedio entre los valores que fija un Hospital Militar de referencia, los valores del Nomenclador de Prácticas Médicas del PAMI y los costos propios de cada prestación. Los valores se revisan y eventualmente se actualizan semestralmente (FUSANA, 2012).

Las Figuras 1 y 2 resumen el proceso en su etapa de facturación y gestión de deudas, respectivamente. Los diagramas muestran el proceso administrativo de facturación y cobro realizado por la AOS en el HNPB, así como la participación de la FUSANA en el proceso. 


\section{Figura 1.}

Etapa de facturación del proceso de recupero de costos del HNPB

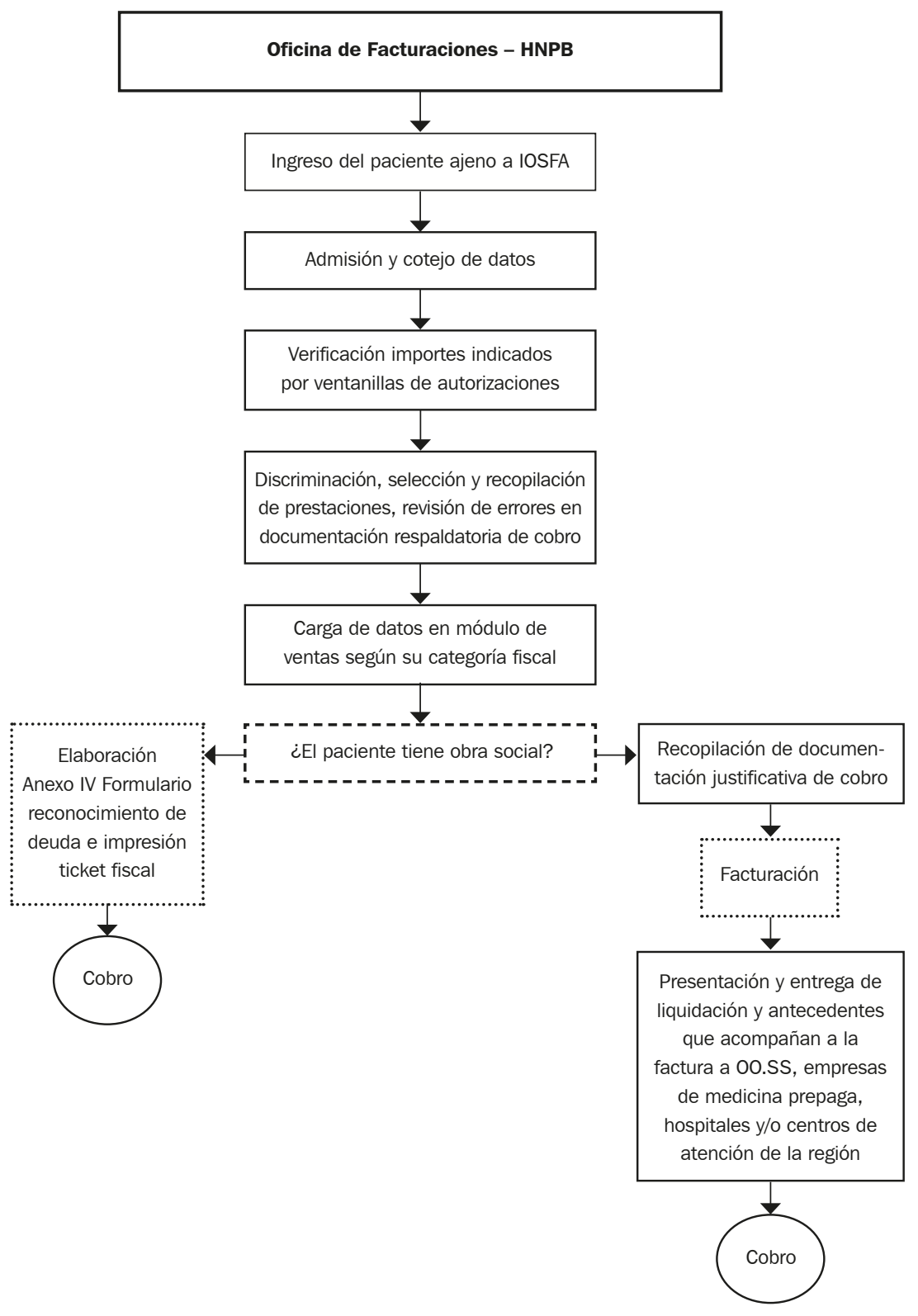




\section{Figura 2.}

Etapa de gestión de deudas del proceso de recupero de costos del HNPB

\section{Oficina de Facturaciones - HNPB}

Fundación de Sanidad Naval

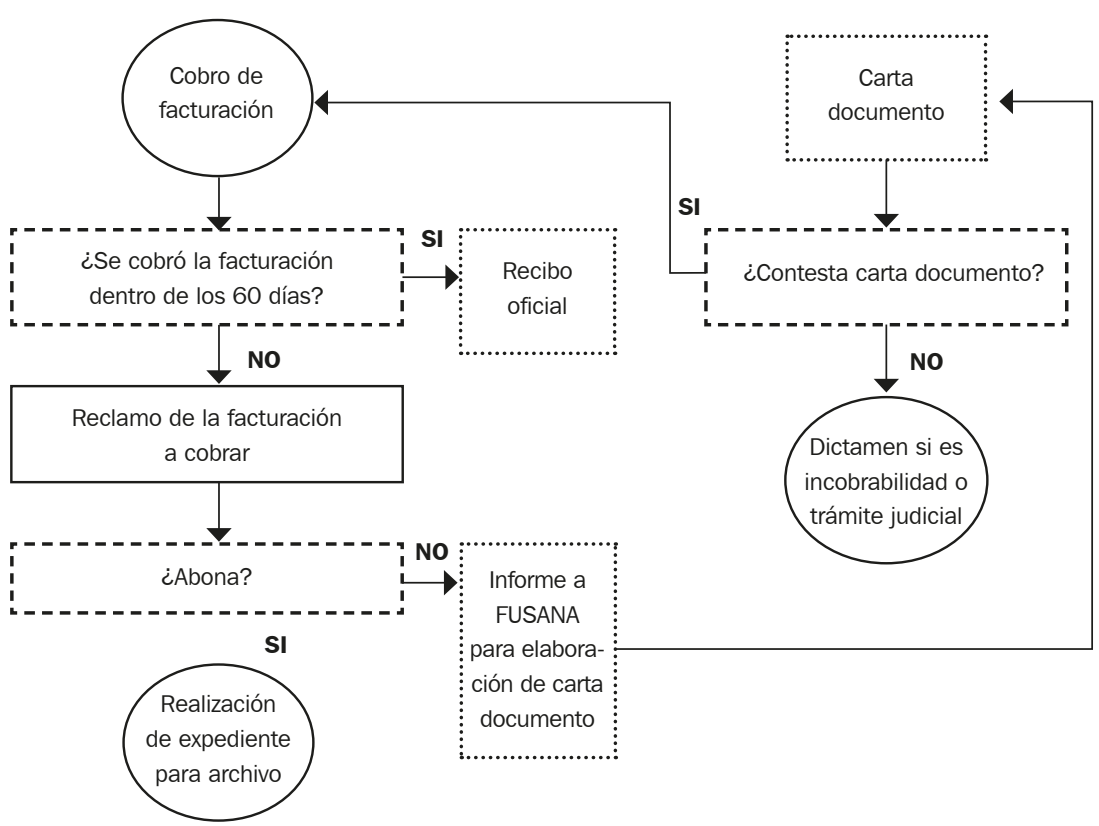




\subsection{RECUPERO DE COSTOS POR PACIENTES INTERNADOS}

NO BENEFICIARIOS DE IOSFA ENTRE 2016-2018

Las 197 internaciones de pacientes no beneficiarios de IOSFA que se registraron durante el período representaron el 2,5\% del total de prestaciones brindadas por el HNPB. La facturación total anual derivada de esas prestaciones tuvo un incremento significativo de un 75,7\% del año 2016 a 2017 , descendiendo notablemente del año 2017 al 2018, ubicándose en un 14\%. El total facturado por este concepto representó entre un 0,80 y un 1,10\% del presupuesto total del HNPB en el periodo, que depende en un 95\% de recursos provenientes de IOSFA (Cuadro 1).

Tanto las prestaciones brindadas a pacientes internados no beneficiarios de IOSFA como los montos facturados se concentran fundamentalmente en tres grandes áreas de atención: cirugías, sala de internación y terapia intensiva (Cuadro 2).

El $47 \%$ de los pacientes internados no beneficiarios de IOSFA poseen cobertura de PAMI, un 31\% son particulares (no poseen cobertura o abonan mediante gasto bolsillo), mientras que un $8 \%$ corresponde a IOMA, 8\% a la ART Provincia y un 4\% a OSPE. Finalmente, el 2\% restante en la categoría "Otros" la integran las Obras Sociales OSDIPP, OSFATLYF, OSGRA, OSPEGAP, Policía Federal y Universal Assistance (Gráfico 1).

El Cuadro 3 resume para cada año del período los montos totales facturados que fueron efectivamente cobrados y los que se encuentran pendientes de pago a noviembre de 2019, así como el índice de incobrabilidad construido en base a dicha información. El índice de incobrabilidad de todo el período por las prestaciones brindadas a pacientes internados no beneficiarios de IOSFA fue de un 20,9\%, notándose un leve descenso en el año 2017 (12,49\%).

En el Cuadro 4, que resume para cada año y según deudor los montos pendientes de facturación, se observa que PAMI, el Hospital Municipal y los pacientes particulares concentran el mayor porcentaje de facturación pendiente de cobro en el período de estudio. Los casos de IOMA, OSGRA y otro Hospital Público (distintos al Municipal y al Interzonal) que poseen montos importantes aún no cobrados corresponden a casos individuales y las mencionadas entidades son poco frecuentes.

Finalmente, en relación a la demora en el cobro de las facturaciones de las prestaciones brindadas a los pacientes internados no beneficiarios de IOSFA que fueron cobradas, se destacan muchos casos de demoras mayores a 6 meses en promedio. En el caso de PAMI, la demora promedio es de 315 días y en el de los pacientes particulares, de 122 días. 


\section{Cuadro 1.}

Facturación total a ajenos IOSFA y recursos presupuestarios

(según fuente de financiamiento) por año (en pesos)

\begin{tabular}{|c|c|c|c|c|}
\hline \multirow[t]{2}{*}{ Año } & \multirow[t]{2}{*}{ Monto Total Facturado AOS } & \multicolumn{3}{|c|}{ Recursos Presupuestarios } \\
\hline & & IOSFA & Pres. Nacional & Total \\
\hline 2016 & \$1.599.185 & \$191.788.152 & $\$ 9.500 .400$ & \$201.288.552 \\
\hline 2017 & $\$ 2.809 .541$ & $\$ 239.735 .190$ & $\$ 11.875 .500$ & \$251.610.690 \\
\hline 2018 & $\$ 3.205 .185$ & $\$ 292.825 .428$ & $\$ 19.200 .000$ & $\$ 312.025 .428$ \\
\hline Total & $\$ 7.613 .912$ & $\$ 724.348 .772$ & $\$ 40.575 .900$ & $\$ 764.924 .672$ \\
\hline
\end{tabular}

Fuente: elaboración propia.

\section{Cuadro 2.}

Prestaciones brindadas y monto facturado según área de internación (en pesos)

\begin{tabular}{lrr}
\hline Área de Internación & Cantidad (\%) & Monto Facturado (\%) \\
\hline Cirugía & $51(25,9 \%)$ & $\$ 1.095 .357(14,4 \%)$ \\
Neonatología & $5(2,5 \%)$ & $\$ 80.366(1,1 \%)$ \\
Sala Común & $106(53,8 \%)$ & $\$ 3.642 .935(47,8 \%)$ \\
Terapia Intensiva & $35(17,8 \%)$ & $\$ 2.795 .253(36,7 \%)$ \\
Total & $\mathbf{1 9 7 ( 1 0 0 \% )}$ & $\mathbf{\$ 7 . 6 1 3 . 9 1 2 ( 1 0 0 \% )}$ \\
\hline
\end{tabular}

Fuente: elaboración propia. 


\section{Gráfico 1.}

Distribución de pacientes según cobertura de salud (2016-2018)

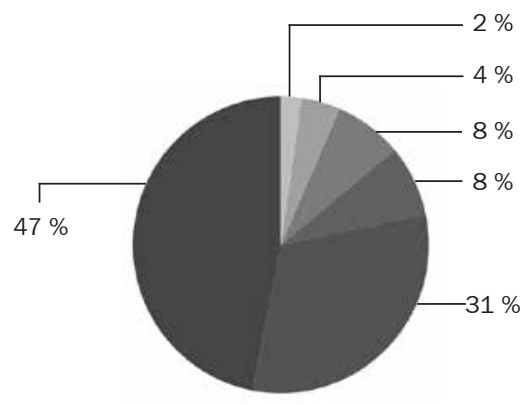

- PAM

Particular

ART Provincia

IOMA

OSPE

Otros

Fuente: elaboración propia

\section{Cuadro 3.}

Montos cobrados, pendientes e índice de incobrabilidad por año (en pesos)

\begin{tabular}{llccc}
\hline Año & Cobrado & $\begin{array}{l}\text { Pendiente a } \\
\text { noviembre 2019 }\end{array}$ & Total & $\begin{array}{l}\text { Índice de } \\
\text { incobrabilidad }\end{array}$ \\
\hline 2016 & $\$ 1.223 .515,07$ & $\$ 375.670,04$ & $\$ 1.599 .185,11$ & $23,49 \%$ \\
2017 & $\$ 2.458 .520,65$ & $\$ 351.020,88$ & $\$ 2.809 .541,53$ & $12,49 \%$ \\
2018 & $\$ 2.348 .992,60$ & $\begin{array}{c}\text { \$ } 356.193,25 \\
\text { Total gral. }\end{array}$ & $\$ 3.205 .185,85$ & $26,71 \%$ \\
& & $\mathbf{\$ 7 . 6 1 3 . 9 1 2 , 4 9}$ & $\mathbf{2 0 , 9 0 \%}$ \\
\hline
\end{tabular}

Fuente: elaboración propia. 


\section{Cuadro 4.}

Montos pendientes de pago por año y actor (en pesos)

\begin{tabular}{|c|c|c|c|}
\hline Deudor & 2016 & 2017 & 2018 \\
\hline Particular & $\$ 26.439(1,7 \%)$ & $\$ 175.165,51(6,2 \%)$ & $\$ 50.105(1,6 \%)$ \\
\hline H. Municipal & $\$ 342.373(21,4 \%)$ & $\$ 105.246,99(3,7 \%)$ & $\$ 249.840(7,8 \%)$ \\
\hline OSGRA & $\$ 6.857(0,4 \%)$ & $\$ 0$ & $\$ 0$ \\
\hline Otro Hospital & $\$ 0$ & $\$ 17.226(0,6 \%)$ & $\$ 0$ \\
\hline Art Provincia & $\$ 0$ & $\$ 53.381(1,9 \%)$ & $\$ 0$ \\
\hline PAMI & $\$ 0$ & $\$ 0$ & $\$ 174.955(5,5 \%)$ \\
\hline IOMA & $\$ 0$ & $\$ 0$ & \$ $381.292(11,9 \%)$ \\
\hline Total & $\$ 375.670(23,5 \%)$ & \$ $351.020(12,5 \%)$ & $\$ 856.193(26,7 \%)$ \\
\hline
\end{tabular}

Fuente: elaboración propia.

\subsection{DIFICULTADES Y POSIBILIDADES DE MEJORA \\ EN EL PROCESO DE RECUPERO DE COSTOS DEL HNPB}

Desde la perspectiva del personal de la AOS se destacaron como problemas que la gestión de deudas se efectúa sin un adecuado basamento en convenios, por medio de comunicaciones no formales de carácter verbal o escrito y sin un orden establecido en el tiempo. Esto genera una acumulación de montos deudores al no cancelarse en tiempo y forma las facturaciones pendientes. Asimismo, la ausencia de base de datos externas, tales como el Sistema Integrado de Información Sanitaria Argentino (SISC), el Portal de Ofertas de Prestadores y Proveedores del PAMI (POPP) o los padrones de Obras Sociales acerca de los pacientes no beneficiarios de IOSFA, dificulta el acceso a la información, ya que no se los puede identificar para conocer su condición y tipo de cobertura de salud. También existen discrepancias con las entidades a quienes se debe facturar sobre las prácticas que éstas cubren y las que no, deviniendo esto en la evasión de los pagos. Asimismo, el atraso en los pagos y la inexistencia de intereses punitorios o resarcitorios ocasiona la pérdida del valor monetario en términos reales en contextos inflacionarios.

Desde el punto de vista de los gestores de las instituciones a las que el HNPB presta servicios, los directores de los prestadores públicos de la región coinciden que un convenio con el HNPB beneficiaría no solo a los pacientes sin cobertura de la ciudad del HNPB, sino también a los que habitan en la región porque podrían utilizarse los servicios no disponibles en el Hospital 
Interzonal. Por su parte, el director regional de PAMI sostiene que la principal ventaja de la celebración de convenios con el HNPB radicaría en aprovechar la proximidad de los beneficiarios de PAMI que residen en la localidad del nosocomio, descomprimiendo la demanda de algunos prestadores de salud, fundamentalmente en determinadas épocas del año. Según el director del Hospital Municipal estarían dadas las condiciones para celebrar un acuerdo marco a nivel regional entre dicho nosocomio, el Hospital Interzonal y el HNPB, estableciendo pautas claras en relación a la atención de los pacientes y el recupero de costos y brindando un beneficio de orden social para la región. Sin embargo, la directora del Hospital Interzonal considera que la cadena de pago puede tornarse difícil entre entidades de distintos niveles de gobierno.

\section{4 discusiōN}

Dada su ubicación geográfica y su nivel de complejidad, el HNPB brinda prestaciones médicas en condiciones de urgencia a pacientes no beneficiarios de IOSFA. El recupero de costos de estas internaciones constituye un problema de gestión económico-financiera para el citado nosocomio. Este problema ha sido estudiando en los hospitales públicos de Argentina y se ha diagnosticado su relevancia, pero no existen antecedentes de su estudio en el caso de hospitales militares.

Se ha observado que el proceso de recupero de costos es un sistema complejo que posee una etapa de facturación y una de gestión de deudas, asentadas sobre reglamentación y normativa interna. El HNPB tiene la posibilidad de recuperar costos por fuera de la Ley 24.156 , teniendo que acudir a una fundación sin fines de lucro como la FUSANA para lograr un mayor aprovechamiento de los recursos y así mitigar el desincentivo al recupero de costos.

Un hallazgo de relevancia es que las prestaciones efectuadas a pacientes no beneficiarios a IOSFA no son significativas en el total de las efectuadas por el nosocomio, como tampoco en relación a los recursos provenientes de IOSFA y del Estado Nacional que financian las actividades. Sin embargo, estos recursos sufren una depreciación real de su valor, dado el alto grado de incobrabilidad y la excesiva demora en la cadena de pagos por parte de las entidades deudoras.

Si bien el HNPB posee un sistema de procesamiento de facturación y cobro de prestaciones, no existen acuerdos formales con los terceros pagadores $u$ otros prestadores públicos, lo que complejiza el proceso inherente al cobro de los servicios brindados. Se encontró que la demora promedio de cobro es 
sumamente elevada, situación que resulta de la ausencia de mecanismos de pago prestablecidos (lineamientos claros de facturación, plazos de presentación de documentación obligatoria de pago, intereses punitorios y/o resarcitorios y demás asuntos de orden jurídico que eviten dilaciones e incobrabilidades).

Dentro del grupo de entidades a las cuales el HNPB brinda prestaciones médicas de internación ante urgencias, el PAMI y los pacientes particulares con y sin capacidad económica concentran casi el $80 \%$ de las facturaciones. Estos últimos, fundamentan la relación con el Hospital Interzonal y el Hospital Municipal, siendo este último la entidad con más alto índice de incobrabilidad durante el periodo.

Ante la internación y posterior atención de los pacientes no beneficiarios de IOSFA, se transfieren recursos del HNPB para subsidiar a pacientes sin cobertura o cubiertos por obras sociales distintas a IOSFA, como por ejemplo PAMI, generando así un fenómeno similar al de los subsidios cruzados desde Ios hospitales públicos de autogestión descentralizada hacia la seguridad social (que se explica mayormente por la diferencia entre los montos del nomenclador y los costos de atención). Sin embargo, el subsidio cruzado se produce aquí en sentido inverso, ya que un actor de la seguridad social (en este caso IOSFA que es el principal financiador del HNPB) financia a instituciones del sector público u otras obras sociales.

Las limitaciones que se encontraron en el presente trabajo están vinculadas a la imposibilidad de acceder a las historias clínicas de los pacientes atendidos, situación que hubiera facilitado una mayor discriminación de las prácticas médicas con respecto a los diagnósticos. Asimismo, no se tuvieron en cuenta los costos hospitalarios asociados a cada una de las prestaciones brindadas. Este aspecto con dificultad se aborda en los estudios de recupero de costos de los hospitales públicos argentinos (Moscoso y Clark, 2010).

La celebración de acuerdos posibilitaría el aprovechamiento de los servicios del HNPB por parte de todos los pacientes de la región no beneficiarios de IOSFA, constituyendo un nicho de oportunidad para la generación de nuevos recursos genuinos para el nosocomio. Futuros trabajos de investigación podrían evaluar en profundidad el potencial beneficio de establecer mecanismos de pago concretos a través de la celebración de distintos tipos de convenios, tales como el modelo de reembolso, modelo integrado o modelo de contrato (Titelman et al., 2000). 


\section{CONCLUSIONES}

El proceso de recupero de costos en los hospitales militares de Argentina permite que estos utilicen capacidad instalada ociosa brindando prestaciones a ciudadanos de la región donde se encuentran localizados que no pertenecen a su población objetivo. Pese a que los recursos económicos obtenidos mediante este mecanismo no serían de relevancia para el financiamiento hospitalario, a diferencia de lo que ocurre en los hospitales públicos provinciales o municipales, es a través de la facturación a terceros, que el nosocomio se relaciona con los diferentes actores del sistema de salud de la región. Por este motivo adquiere relevancia incrementar la participación y el protagonismo de este proceso administrativo. En este sentido, la existencia de contratos de prestación entre la institución y los restantes prestadores públicos de la región, así como con agentes financiadores permitiría un mejor aprovechamiento de la capacidad remanente en los hospitales militares en beneficio del conjunto de la sociedad.

\footnotetext{
Agradecimientos

Los autores agradecemos los valiosos comentarios de la Mag. Marcela Clark y el Esp. Gastón De Leo quienes integraron el jurado del trabajo final de la Especialización en Economía y Gestión de los Servicios de Salud del Departamento de Economía (Universidad Nacional del Sur) del cual se desprende este artículo. También agradecemos las acertadas sugerencias de dos revisores anónimos.
} 


\section{NOTAS}

${ }^{1}$ Existen otras dos situaciones que originan subsidios cruzados entre subsistemas: i) cuando beneficiarios de las obras sociales las utilizan para acceder a la cobertura de empresas de medicina prepaga (problema conocido como descreme de las obras sociales) y ii) cuando individuos tienen más de una obra social o cuando teniendo obra social contratan un seguro privado en una empresa de medicina prepaga (problema conocido como doble cobertura formal).

2 La figura de los hospitales públicos de gestión descentralizada aparece en la década de los años '90 para mejorar la gestión hospitalaria otorgándole a los hospitales públicos la posibilidad de cobrar las prestaciones demandadas por beneficiarios de obras sociales a través de un sistema de débito automático. Este mecanismo de recupero de costos al asegurar el cobro mejoraba la eficiencia de la recaudación de ingresos genuinos y contribuía a mitigar el problema de los subsidios cruzados (Moscoso y Modarelli, 2009). ${ }^{3}$ Las normativas revisadas fueron las siguientes: de los Sistemas de Control del Sector Público Nacional del año 1992, ii) Decreto PEN N ${ }^{\circ}$ 2.629 del año 1992, iii) Convenio de concesión de capacidades remanentes entre la Dirección General de Salud de la Armada Argentina y la FUSANA del año 2012, iv) Manual de Gestión y Ejecución Presupuestaria - Manejo de Fondos, complementario del R.A-8-001 - Reglamento de Administración Naval de la Armada Argentina del año 1978, v) Manual de Gestión y Ejecución Presupuestaria - Manejo de Fondos, complementario del R.A-8-001 - Reglamento de Administración Naval de la Armada Argentina del año 1978, vi) Resolución General N 7/2015 de la Inspección General de Justicia (I.G.J) referente a fundaciones sin fines de lucro, vii) Disposición de Salud de la FUSANA No 13 referente a normas de procedimiento sobre regulación sobre manejo y contabilidad de fondos del año 2016 y viii) Orden Militar del Director General del HNPB N $70 / 17$ sobre tarifas de las prestaciones brindadas a pacientes no beneficiarios de IOSFA del año 2017.

\section{BIBLIOGRAFÍA}

Aleixechuk C., Clark M. y Temporelli, K. (2013). "Formas de pago y subsidios cruzados en los hospitales públicos: el caso del convenio capitado del Hospital "Dr. Leónidas Lucero» de la ciudad de Bahía Blanca con PAMl». Documentos y Aportes en Administración Pública y Gestión Estatal; 1(21), pp 125-141. https://doi.org/10.14409/da.v1i21.4151.

Belló M., Becerril-Montekio VM. (2011). "Sistema de salud de Argentina». Salud Publica Mex;53 supl 2, pp S96-S108.

Boletín Oficial de la República Argentina № 27.503 (1992). Ley 24.156 (1992), publicado el 29 de octubre de 1992.

Boletín Oficial de la República Argentina № 27.546 (1992). Decreto de Necesidad y Urgencia № 2.629 (1992), publicado el 31 de diciembre de 1992.

Boletín Oficial de la República Argentina № 29.510 (2000). Decreto de Necesidad y Urgencia $N^{\circ} 939$ (2000), publicado el 24 de octubre de 2000. 
Boletín Oficial de la República Argentina № 33.183 (2015). Resolución General № 7 de la Inspección General de Justicia, publicado el 31 de julio de 2015.

Cetrángolo 0. (2014). «Financiamiento fragmentado, cobertura desigual y falta de equidad en el Sistema de Salud Argentino". Revista de Economía Política de Buenos Aires. [acceso: 20/06/2019]; 8 (13), pp 45-183. Disponible en: http://ojs.econ.uba.ar/index.php/REPBA/ article/view/785/1431

FUSANA (2016). Disposición de Salud No 13 de la Fundación Sanidad Naval Argentina Disponible en: http://www.fusana.org.ar/programa-de-integridad-de-fusana/

(2012). Convenio de concesión de capacidades remanentes entre la Dirección General de Salud de la Armada Argentina y la Fundación Sanidad Naval Argentina (FUSANA). Octubre de 2012

Tobar F, Olaviaga S, Solano R (2012). Complejidad y fragmentación: las mayores enfermedades del sistema sanitario argentino. Documento de Políticas Públicas. 2012 [acceso: 25/06/2019]; № 108. Buenos Aires: CIPPEC. Disponible en: https://www.fmed. uba.ar/sites/default/files/2018-03/108\%20DPP\%20Salud\%2C\%20Complejidad\%20y\%20 fragmentacion\%2C\%20Tobar\%2C\%200laviaga\%20y\%20Solano\%2C\%202012\%5B1\%5D.pdf

Moscoso, N. y Clark, M. (2010). "Relevancia de la Estimación de Costos para la Gestión Hospitalaria». Documentos y Aportes en Administración Pública y Gestión Estatal, 1(15), 29-41. https://doi.org/10.14409/da.v1i15.1258

Moscoso N. y Modarelli R (2009). "Descentralización en Salud: Marco Conceptual y Políticas Públicas en Argentina». Ciencias Económicas, 27(2),155-168.

Titelman, D. y Uthoff, A. (compiladores) (2000). Ensayo sobre el financiamiento de la seguridad social en salud. Los casos de Estados Unidos, Canadá, Argentina, Chile y Colombia. Comisión Económica para América Latina y El Caribe (CEPAL) y Fondo de Cultura Económica de Chile (FCE). 